\title{
Elevación transitoria del ST, angina variante de Prinzmetal
}

\author{
Transitory ST elevation, Prinzmetal angina
}

\author{
Diego Echeverri Marín ${ }^{1 \otimes}$, Hernán Darío Aguirre ${ }^{2 \otimes}$ Researchgate, Juan David Ramírez $^{3 \otimes}$ \\ 1. Medico, Internista. Universidad de La Sabana, Bogotá, Colombia. \\ 2. Médico Internista. Clínica Universitaria Bolivariana/Hospital General de Medellín, Colombia. \\ 3. Médico, Cardiólogo - Electrofisiólogo. Clínica CardioVid. Medellín, Antioquia.
}

Este trabajo cumple con las siguientes características editoriales

Fecha correspondencia: Recibido: mayo 4 de 2015. Revisado: febrero 29 de 2016. Aceptado: marzo 18 de 2016.

Forma de citar:

Echeverri-Marín D, Aguirre HD, Ramírez JD. Elevación transitoria del ST, angina variante de Prinzmetal. Rev CES Med 2016; 30(1): 107-113.

Open access

(C) Derecho de autor Licencia creative commons

Ética de publicaciones

Revisión por pares

Gestión por Open Journal System

ISSN 0129-8705

ISSN-e 2215-9177

Comparte

\section{CES MEDICINA}

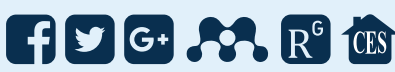

\section{Resumen}

La angina variante de Prinzmetal es una enfermedad poco frecuente en Colombia, pero con alto riesgo de complicaciones sino se diagnostica e inicia su manejo precozmente. Así mismo, es una de las causas de elevación del segmento ST en el electrocardiograma en pacientes con dolor torácico. Se expone el caso de una paciente con enfermedad vasoespástica y cuya presentación clínica simuló una infarto agudo de miocardio y luego de realizar los estudios diagnósticos se pudo descartar éste y dar el tratamiento adecuado.

Palabras clave: Angina variante de Prinzmetal, Dolor torácico, Enfermedad vasoespástica.

\begin{abstract}
Prinzmetal variant angina is an uncommon disease in Colombia, it have high risk of complications if is not diagnosed early and a rapid treatment starter. Also, it is one of the causes of ST segment elevation on the electrocardiogram in patients with chest pain in the emergency department. We describe the case of a patient with vasospastic disease whose clinical presentation simulates a heart attack and after diagnostic studies that allowed dismiss this and, the proper treatment was given.
\end{abstract}

Keywords: Prinzmetal's variant angina, Chest pain, Vasoespastic disease.

\section{Introduction}

La angina de etiología vasoespástica fue descrita Inicialmente por el Dr. Myron Prinzmetal en 1959. En su primer reporte, el Dr. Prinzmetal y su equipo reportan una serie de 35 casos haciendo referencia a ésta como una variante a la angina clasica, dadas las características clínicas atípicas de su presentación (1).

En estos pacientes se realizó coronariografía y en la mayoría no se demostró estenosis coronaria significativa, además, reportaron que a pesar de llevar a nueve de estos pacientes a revascularización quirúrgica, persistieron con el síntoma, pero apreciaron mejoría tras la administración de nifedipino. Con este hallazgo y tratamiento inicial abrieron la puerta al manejo médico de estos pacientes y propusieron factores neurohumorales como posibles mecanismos implicados en la fisiopatología de la enfermedad (1). 
Se conoce este tipo de angina como una enfermedad en la cual existe una vasoconstricción coronaria repentina y excesiva, que produce una reducción transitoria del flujo sanguíneo miocárdico y de acuerdo a la duración del mismo, puede conllevar a isquemia miocárdica, incluso progresar a necrosis del tejido.
Al día de hoy se conoce este tipo de angina como una enfermedad en la cual existe una vasoconstricción coronaria repentina y excesiva, que produce una reducción transitoria del flujo sanguíneo miocárdico y de acuerdo a la duración del mismo, puede conllevar a isquemia miocárdica, incluso progresar a necrosis del tejido. La alteración en el flujo coronario comúnmente está acompañado de una elevación transitoria del segmento ST en las derivaciones representantes del territorio implicado (2). Aunque el espasmo coronario se produce principalmente en las arterias epicárdicas que transcurren por la superficie del corazón, también puede ocurrir en la microvasculatura coronaria (2).

Su incidencia en Colombia es desconocida dada la poca cantidad de casos, situación contraria ocurre en Japón, donde es tres veces más común que en Europa (3). Por la alta prevalencia de la enfermedad en algunas zonas geográficas a nivel del mundo se sospecha que esta enfermedad tenga alguna relación con factores genéticos o raciales $(4,5)$.

\section{Descripción del caso}

Se trataba de una paciente femenina de 34 años de edad quien consultó al servicio de urgencias por dos episodios de dolor torácico opresivo. El primer episodio inició en horas de la noche del día anterior a la consulta, mientras se encontraba en reposo y relataba un dolor de intensidad moderada, no irradiado, asociado a sensación de "adormecimiento" del miembro superior izquierdo y con duración aproximadamente de cinco horas, el cual se auto-limitó sin medicación en horas de la madrugada del día siguiente.

Cuatro horas más tarde presentó un nuevo episodio de mayor intensidad, motivo por el cual consultó al servicio de urgencias, donde se procedió a la realización de electrocardiograma convencional (ECG) (figura 1).

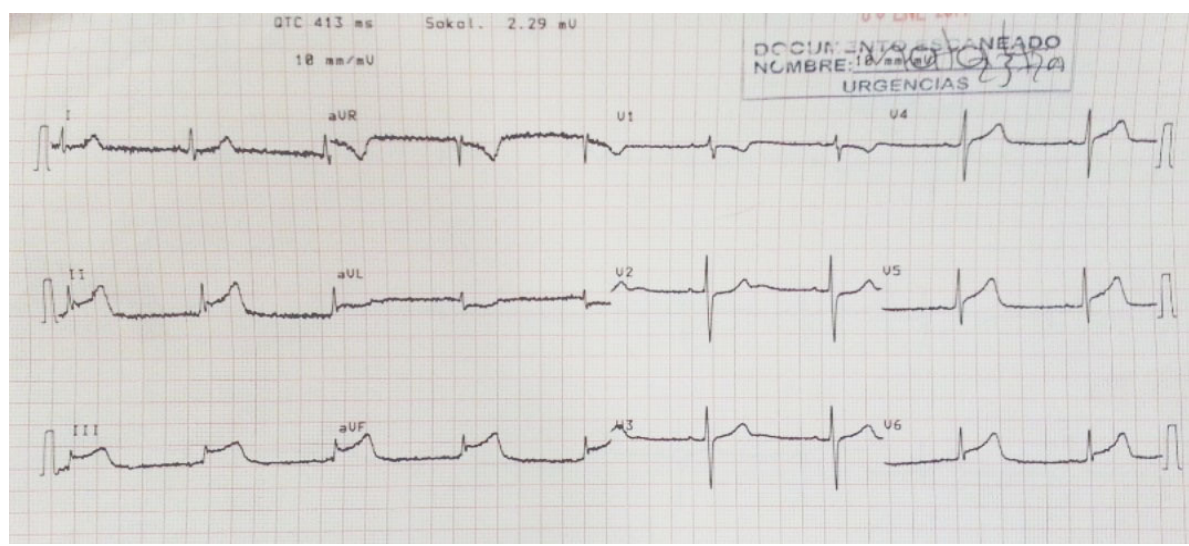

Figura 1. Electrocardiograma convencional. Elevación del segmento ST en territorio inferior y representación eléctrica en pared lateral baja

Al encontrar elevación cóncava del segmento ST en cara inferior con compromiso de la pared lateral, se consideró el diagnóstico de síndrome coronario agudo con elevación de ST de cara inferior. Se inició manejo con heparina no fraccionada, inhibidor del 2YP12, ácido acetilsalicílico y estatina y se trasladó a unidad de cuidados intensivos.

Allí se realizó electrocardiograma de control a los 45 minutos luego del primer ECG (figura 2), aunque en ese momento la paciente no relataba dolor. En dicho examen se 
Ante la sospecha de vasoespasmo coronario se dio tratamiento médico con calcio-antagonista tipo diltiazem y se continuó con ácido acetilsalicílico 100 mg/día y estatina, siendo de gran impacto el uso de calcioantagonista sobre otros grupos farmacológicos. evidenció aplanamiento de la elevación del segmento ST en más del 70 \%. Se tomó medición de troponina I ultrasensible, con valor de $0,4 \mathrm{ng} / \mathrm{ml}$ (positiva para el valor de referencia del laboratorio).

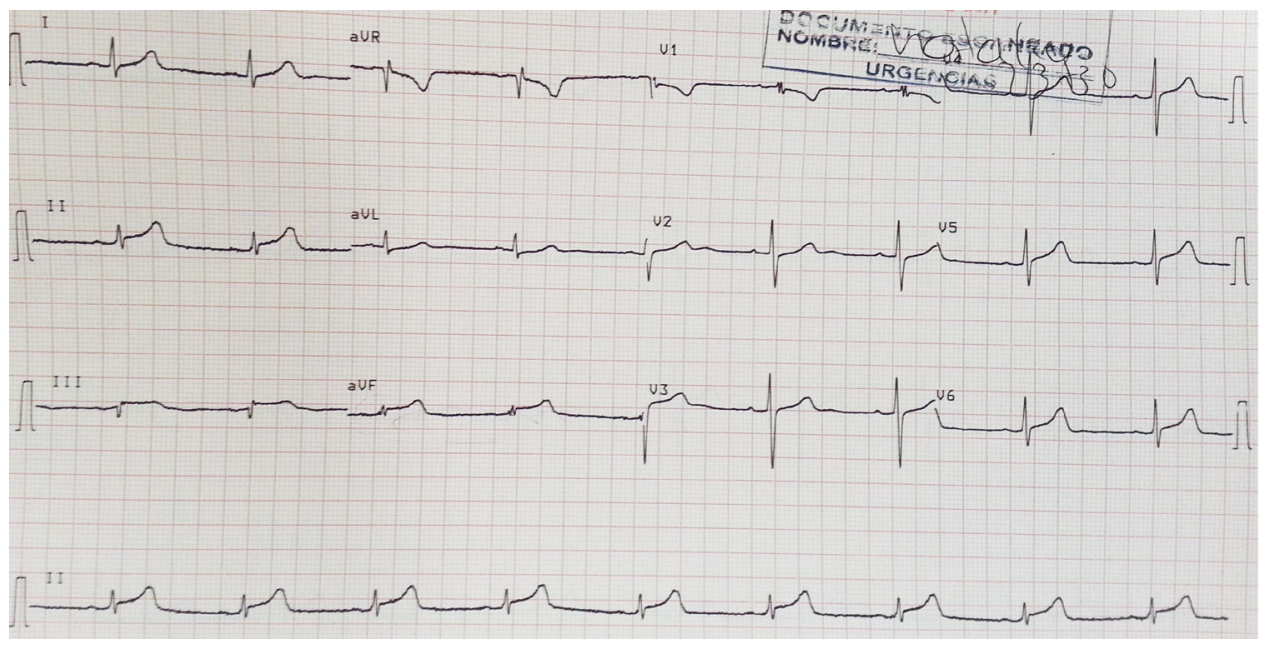

Figura 2. Electrocardiograma convencional. Aplanamiento de más del $70 \%$ de la elevación del segmento ST previa

Se realizó ecocardiograma transtorácico en el que se encontró función biventricular normal sin alteraciones de la contractilidad. No había alteraciones en radiografía de tórax.

Al día siguiente se llevó a coronariografía, en donde no se evidenciaron lesiones en las arterias subepicárdicas y con ventriculograma normal (figura 3). Ante la sospecha de vasoespasmo coronario se dio tratamiento médico con calcio-antagonista tipo diltiazem y se continuó con ácido acetilsalicílico 100 mg/día y estatina, siendo de gran impacto el uso de calcioantagonista sobre otros grupos farmacológicos, ya que se logró controlar síntomas y disminuir su riesgo de morbimortalidad cardiovascular.
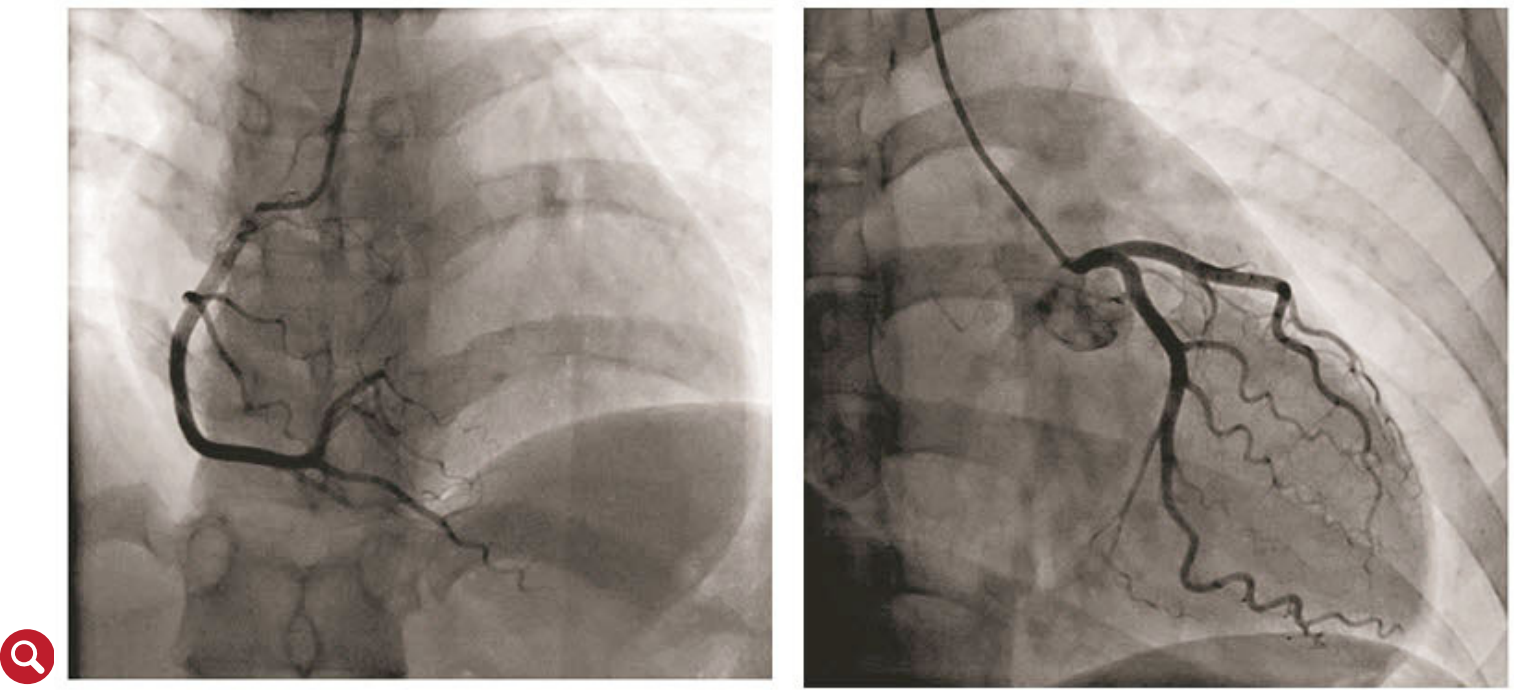

Figura 3. Coronariografía. Sin lesiones en arterias subepicárdicas 
Como criterios definitorios se consideran: presencia en el ECG de elevación o depresión del segmento ST > 0,1 $\mathrm{mV}$ durante el episodio de dolor torácico; hallazgos de estenosis coronaria angiográfica > $90 \%$ inducida por medicamento vasoespástico o test de hiperventilación.
Se decide no llevar a nueva coronariografia para inducción de vasoespasmo, pues se consideró que era mayor el riesgo que el beneficio y que frente al parámetro de costo/utilidad era preferible iniciar la terapia con calcioantagonistas.

La paciente permaneció sin nuevos episodios anginosos durante la hospitalización y a las 72 horas de su consulta se dio egreso hospitalario.

\section{Discusión}

La angina variante de Prinzmetal se observa principalmente en los pacientes mayores de 40 años, principalmente de género masculino; ambos elementos discordantes con la paciente descrita, aunque se debe recordar que se aprecia aumento de su frecuencia en las mujeres postmenopáusicas. Se presenta principalmente durante el reposo y no se empeora con la actividad física (2).

Este tipo de angina ha presentado disminución en la incidencia debido al uso extendido de calcio-antagonistas ( $\underline{3})$, hecho que se evidenció con mayor claridad en Japón desde el 2003 (ㅁ).

En Europa se ha reportado una incidencia que va desde el $16 \%$ en pacientes con infarto agudo de miocardio, sin lesiones estenóticas en la arteriografía y test de inducción de vasoespasmo coronario positivo con ergonovina (7), hasta porcentajes tan altas como del $49 \%$ en otras publicaciones (8).

La asociación de la angina con el esfuerzo físico difiere de la enfermedad coronaria ateroesclerótica, uno de los diagnósticos diferenciales más importantes de realizar, ya que la angina ocurre predominantemente en horas de la noche y madrugada y durante periodos de reposo físico (9), situación similar a la de la paciente descrita.

Los criterios diagnósticos son los establecidos en las Guías de angina vasoespástica de la Sociedad Japonesa de Circulación publicadas en el 2008 (2), aunque cabe resaltar que no son ampliamente aceptadas en la actualidad por todas las sociedades de cardiología.

Como criterios definitorios de angina vasoespástica de Prinzmetal se considera a los pacientes que presenten: presencia en el ECG de elevación o depresión del segmento ST > 0,1 mV durante el episodio de dolor torácico; hallazgos de estenosis coronaria angiográfica $>90 \%$ inducida por medicamento vasoespástico o test de hiperventilación.

Un caso sospechoso es aquel con angina que desaparece rápidamente tras la administración de un nitrato, más una de las siguientes: aparición en reposo, particularmente en la noche o el amanecer; tolerancia variable al ejercicio durante el día, dolor inducido por episodios de hiperventilación, y, los ataques son suprimidos por medicamentos calcio-antagonistas, pero no por beta-bloqueadores.

La etiología es multifactorial en la que los factores genéticos, tóxicos (tabaquismo, alcohol) (10,11), medicamentos como los triptanes y sustancias quimiotácticas $(12,13)$, juegan un papel muy importante en la génesis y como precipitantes de los eventos.

Se han establecido diferentes polimorfismos genéticos implicados en la fisiopatología de la enfermedad, más específicamente en la codificación de la enzima óxido 
Para la prevención de nuevos eventos los calcio-antagonistas han sido el pilar recomendado. nítrico sintetasa $(\underline{9}, 14,15)$, los cuales conllevan a una disfunción endotelial. Así mismo, alteraciones en la codificación de la Rho kinasa, enzima independiente de calcio intracelular para la relajación muscular, llevando a disfunción a nivel del músculo liso (16).

La ateroesclerosis está implicada en fisiopatología de la enfermedad, dado que el espasmo coronario se presenta en los sitios donde se encuentran las placas ateroescleróticas, muchas de ellas tan pequeñas que solo se evidencian en ultrasonografía intracoronaria (17).

En cuanto al manejo médico, la prevención es el pilar del tratamiento. El abandono del consumo de tabaco es fundamental, así como el control de las entidades que aumentan el riesgo cardiovascular, como la hipertensión arterial, diabetes mellitus, dislipidemia y sobrepeso $(\underline{2}, \underline{10})$.

El tratamiento farmacológico difiere en cuanto al momento de presentación de la enfermedad, dado que en el momento agudo se prefiere el manejo con nitratos sublinguales que los calciantagonistas, los cuales se usan a pesar de no tener fuerte evidencia $(\underline{2}, \underline{18}, \underline{19})$.

En el manejo farmacológico para la prevención de nuevos eventos los calcio-antagonistas han sido el pilar recomendado para el manejo convencional $(1,2,20)$ y no se han demostrado diferencias significativas entre los de corta o larga acción (21). Se observa una tendencia a la disminución de eventos inducidos y espontáneos con los calcioantagonistas cuando se compara con los nitratos orales (22). Se podría considerar a los betabloqueadores como manejo conjunto o coadyuvante al tratamiento con los calcio-antagonistas, pero no como monoterapia (23).

En cuanto al papel de las estatinas en el manejo de la enfermedad vasoespástica, especialmente la fluvastatina, se evidencia una reducción hasta del 30 \% de eventos coronarios vasoespásticos en los pacientes a quienes se les añadió al tratamiento convencional (nifedipino 20-40 mg o diltiazem 100-200 mg) (24).

Se recomienda el manejo con ácido acetílsalicílico 75-300 mg/día, dada la activación de la vía primaria de la coagulación. No se recomienda dosis más altas por el efecto que existe en la inhibicion de produccion de prostanglandinas, esenciales en la regulación endotelial (2).

El tratamiento percutáneo con angioplastia o stent está muy limitado, dado los malos resultados evidenciados y se reserva para casos muy severos (25).

El curso de la enfermedad depende del tiempo y el compromiso de las arterias coronarias implicadas, pudiendo llevar a arritmias fatales, infarto, bloqueos AV transitorios, entre otras complicaciones. El pronóstico de la enfermedad es bueno y depende del número de vasos implicados y la función ventricular del paciente (26).

\section{Conclusión}

La angina variante de Prinzmetal continúa siendo una rara presentación de enfermedad coronaria, además, poseemos poco conocimiento de su epidemiología en el mundo occidental, debido al incremental uso de calcio antagonistas para otras enfermedades cardiovasculares, con lo cual se termina ofreciendo una profilaxis primaria para los pacientes que sufrirían de este tipo de angina. 


\section{Conflicto de interes}

Los autores no presentan conflicto de interés alguno.

\section{Bibliografía}

1. Prinzmetal M, Kennamer R, Merliss R, Wada T, Bort N Angina pectoris. I. A variant form of angina pectoris; preliminary report. Am J Med. 1959 Sep;27:375-88. link

2. JCS Joint Working Group. Guidelines for diagnosis and treatment of patients with vasospastic angina (coronary spastic angina) (JCS 2008): digest version. Circ J. 2010 Aug;74(8):1745-62. link

3. Shozo S, Yasuhiro S, Tomoki S. Recommendation for establishment of guidelines for Prinzmetal's variant angina and vasospastic angina in the USA and Europe. J Cardiol Cases. 2012 Nov 6:5:161-162. link

4. Beltrame JF, Sasayama S, Maseri A.Racial heterogeneity in coronary artery vasomotor reactivity: differences between Japanese and Caucasian patients. J Am Coll Cardiol. 1999 May;33(6):1442-52. link

5. Pristipino C, Beltrame JF, Finocchiaro ML, Hattori R, Fujita M, Mongiardo R, et. Major racial differences in coronary constrictor response between Japanese and Caucasians with recent myocardial infarction. Circulation. 2000 Mar 14;101(10):1102-8. link

6. Sueda S, Kohno H, Fukuda H, Uraoka T. Did the widespread use of long-acting calcium antagonists decrease the occurrence of variant angina? Chest. 2003 Dec;124(6):2074-8. link

7. Da Costa A, Isaaz K, Faure E, Mourot S, Cerisier A, Lamaud M.Clinical characteristics, aetiological factors and long-term prognosis of myocardial infarction with an absolutely normal coronary angiogram; a 3-year follow-up study of 91 patients. Eur Heart J. 2001 Aug;22(16):1459-65. link

8. Ong P, Athanasiadis A, Hill S, Vogelsberg H, Voehringer M, Sechtem U. Coronary artery spasm as a frequent cause of acute coronary syndrome: the CASPAR (coronary artery spasm in patients with acute coronary syndrome) study. J Am Coll Cardiol 2008;52:523-7. link

9. Yasue H, Kugiyama K. Coronary spasm: Clinical features and pathogenesis. Intern Med 1997; 36: 760 - 765. link

10. Nobuyoshi M, Abe, Nosaka H, Kimura T, Yokoi H, Hamasaki N, et al. Statistical analysis of clinical risk factors for coronary spasm: identification of the most important. Am Heart J 1992; 124:32. link

11. Takaoka K, Yoshimura M, Ogawa H, Kugiyama K, Nakayama M, Shimasaki Y, et al. Comparison of the risk factors for coronary spasm with those for organic stenosis in Japanese population: role of cigarette smoking. Int J Cardiol 2000; 72:121. link

12. Maseri A, Lanza G. Fluorouracil-induced coronary artery spasm. Am J Med 2001; 111:326. link

13. Wasson S, Jayam VK. Coronary vasospasm and myocardial infarction induced by oral sumatriptan. Clin Neuropharmacol 2004; 27:198. link 
14. Gluek J, Valdés A, Bowe D, Wang P. The endothelial nitric oxide synthase T-786c mutation, a treatable etiology of Prinzmetal's angina. Translational Research. Volume 162, Number 1. link

15. Smith KJ, Chadburn AJ, Adomaviciene A, Minoretti P, Vignali L, Emanuele E, et. Coronary spasm and acute myocardial infarction due to a mutation (V734I) in the nucleotide binding domain 1 of ABCC9.Int J Cardiol. 2013 Oct 9;168(4):3506-13. link

16. Amano M, Ito M, Kimura K, Fukata Y, Chihara K, Nakano T, et al, Phosphorylation and activation of myosin by Rho-associated kinase (Rho-kinase).J Biol Chem. 1996 Aug 23;271(34):20246-9. ink

17. Ozaki Y, Keane D, Serruys PW. Progression and regression of coronary stenosis in the long-term follow-up of vasospastic angina. Circulation. 1995; 92: 2446-2456. link

18. Pepine CJ, Feldman RL, Conti CR. Action of intracoronary nitroglycerin in refractory coronary artery spasm. Circulation 1982; 65: 411. link

19. Kugiyama K, Ohgushi M, Sugiyama S, Motoyama T, Kawano H, Hirashima O, Yasue $\mathrm{H}$. Supersensitive dilator response to nitroglycerin but not to atrial natriuretic peptide in spastic coronary arteries in coronary spastic angina. Am J Cardiol 1997;79:606-610. link

20. Opie LH. Calcium channel antagonists in the management of anginal syndromes: changing concepts in relation to the role of coronary vasospasm.Prog Cardiovasc Dis. 1996 Jan-Feb;38(4):291-314. link

21. Ardissino D., Savonitto S, Mussini A, Zanini P, Rolla A, Barberis P, et al. Felodipine (once daily) versus nifedipine (four times daily) for Prinzmetal's angina pectoris. American Journal of Cardiology 1991 68:17 (1587-1592). link

22. Lombardi M, Morales MA, Michelassi C, Moscarelli E, Distante A, L'Abbate A. Efficacy of isosorbide-5-mononitrate versus nifedipine in preventing spontaneous and ergonovine-induced myocardial ischaemia. A double-blind, placebo-controlled study. Eur Heart J. 1993 Jun;14(6):845-51. Link

23. Cohn PF. Concomitant use of nitrates, calcium channel blockers, and beta blockers for optimal antianginal therapy. Clin Cardiol. 1994 Aug;17(8):415-21. link

24. Yasue H, Mizuno Y, Harada E, Itoh T, Nakagawa H, Nakayama M, et al. Effects of a 3-hydroxy-3-methylglutaryl coenzyme A reductase inhibitor, fluvastatin, on coronary spasm after withdrawal of calcium-channel blockers. J Am Coll Cardiol. 2008 May 6;51(18):1742-8. link

25. Tanabe Y, Itoh E, Suzuki K, Ito M, Hosaka Y, Nakagawa I, et al. Limited role of coronary angioplasty and stenting in coronary spastic angina with organic stenosis. J Am Coll Cardiol. 2002 Apr 3;39(7):1120-6. link

26. Waters DD, Miller DD, Szlachcic J, Bouchard A, Méthé M, Kreeft J, et al. Factors influencing the long-term prognosis of treated patients with variant angina. Circulation. 1983 Aug;68(2):258-65. link 